\title{
AS MULTIFACETAS DO EXCESSO DE PESO NA CRIANÇA: UMA REVISÃO SISTEMÁTICA
}

\author{
THE MULTIPLE FACETS OF OVERWEIGHT IN CHILD: A SYSTEMATIC REVIEW
}

\section{Maria Juliete Maia Gomes ${ }^{\mathrm{a}^{*}}$, Ellany Gurgel Cosme do Nascimento ${ }^{b^{*}}$}

\author{
ajuliete_gomesenfermagem@hotmail.com, bellanygurgel@ hotmail.com \\ *Universidade do Estado do Rio Grande do Norte - Pau dos Ferros (RN), Brasil
}

Data de recebimento do artigo: 23/03/2014 Data de aceite do artigo: 22/01/2015

\section{RESUMO}

Introduçáo: A obesidade é um tema desafiador na literatura científica e justifica-se pelo aumento exponencial da prevalência em todas as camadas da sociedade. Objetivos: Identificar e revisar como as publicaçôes discutem os fatores influenciadores no aumento de peso nas crianças e assim propor possíveis intervençóes de enfrentamento. Materiais e Métodos: Revisão sistemática das publicaçôes mais impactantes sobre os determinantes do excesso de peso na população infantil, utilizando as bases de dados MEDLINE/Pubmed e a SciELO-BR. Quanto aos critérios, foram eleitos os últimos cinco anos de artigos de livre acesso, pesquisas com ambos os sexos de 0 a 12 anos de idade, de modo que, para confirmação da qualidade das informações, foi trabalhado com um checklist/roteiro norteador para organização e classificação das ideias dos autores. Resultado: Foram selecionados 44 artigos, apresentando-se em três categorias: obesidade infantil e serviço de saúde; obesidade infantil e hábitos familiares; obesidade infantil e ambiente escolar. Conclusão: $\mathrm{O}$ estudo permitiu apontar a situação do excesso de peso na população infantil no Brasil e em alguns países desenvolvidos, no período de cinco anos. O excesso de peso na criança é um desafio constante e real, desse modo, vislumbramos que a instituição educacional, o serviço de saúde e a família caracterizam-se como uma tríade basilar no diagnóstico, tratamento e prevenção da obesidade infantil, tendo em vista ser uma doença complexa, crônica e que exige esforço mútuo dos diversos setores sociais, para que se possa buscar a resolução nas intervençóes de enfrentamento desse grave problema de Saúde Pública.

Palavras-chave: Obesidade; comportamento alimentar; hiperfagia; infância; escolares.

\section{ABSTRACT}

Introduction: Obesity is a challenging topic in scientific literature; justified by the exponential increase in prevalence in all layers of society. Objective: Identify and review how the published literature discuss the factors that influence the weight gain in children, then suggest possible interventions. Materials and Methods: It was made a systematic review of the most cited publications about the determiners of the overweight in children, using the databases MEDLINE/Pubmed and SciELO-BR. About the criteria, it was chosen some articles of free access published on the last 5 years, with researches on both sexes from 0 to 12 years old. To reaffirm the quality of the information collected, it was made a checklist/guide to organize and classify the authors' ideas. Results: This review consists of 44 articles selected, which were categorized in three topics: child obesity and healthcare; child obesity and family habits; child obesity and school environment. Conclusion: The study allowed us to point out the overweight in children's populations in Brazil and in some developed countries within the period of 5 years. Overweight in children is a real and constant challenge, therefore, it is noticeable that the educational institution, the healthcare and the family are essential to the diagnosis, treatment and prevention of child obesity, since it is a complex chronicle disease that requires a mutual effort of the different social spheres aiming to face, by intervention, this grave public health problem.

Keywords: Obesity; feeding behavior; hyperphagia; childhood; schools. 


\section{Introdução}

A obesidade é um tema recorrente na literatura científica da atualidade. Isso se deve, em grande parte, ao aumento exponencial da prevalência dessa condiçáo em todas as camadas da sociedade. $\mathrm{O}$ aumento da popularidade desse tema fez também com que assuntos associados a ele emergissem e ganhassem maior notabilidade em publicaçōes na área da saúde $e^{1,35,40,43}$.

A melhoria das condiçóes de vida, a maior cobertura de saúde e o declínio da fecundidade favoreceram a redução da desnutrição no país. Em contrapartida, a urbanização e seu impacto nos padróes de alimentação e atividade física contribuíram para a evolução do excesso de peso e, consequentemente, para as mudanças dos indicadores nutricionais. Essa inversão, num intervalo relativamente curto, coloca a obesidade como um dos problemas prioritários para o campo da saúde pública no Brasil ${ }^{2,42}$.

Estudos epidemiológicos do World Health Organization $^{3}$ e do Instituto Brasileiro de Geografia e Estatística (IBGE) mostram que desde a década de 1970, o número de escolares brasileiros com sobrepeso triplicou e, atualmente, existem mais escolares com excesso de peso do que desnutridos ${ }^{4,32}$.

Estudos realizados em algumas cidades brasileiras mostram que o sobrepeso e a obesidade já atingem 30\% ou mais das crianças e adolescentes. Recife foi uma das cidades nas quais se chegou à conclusão que, dos escolares avaliados, $35 \%$ tinham excesso de peso 5 .

A obesidade infantil e o excesso de peso na infância e na adolescência representam um grande problema de saúde pública mundial, e preocupam cada vez mais seus órgãos responsáveis, devido ao crescimento acelerado da sua prevalência, e ao seu aparecimento como uma questão de importantes repercussóes em aspectos biológicos, sociais, psicológicos e culturais ${ }^{6}$.

Estima-se que, a nível mundial, existam aproximadamente 150 milhóes de escolares com excesso de peso, e quase 45 milhóes destes são obesos ${ }^{7}$. Tem-se que a formação dos hábitos alimentares se inicia na infância, visto que, quando esses hábitos são formados de maneira incorreta, acarretam o risco da criança se tornar obesa na adolescência, representando até 75\% de chances, sendo que na vida adulta pode representar até $40 \% 0^{1,5,12}$.

As consequências da obesidade infantil podem ser vistas em diversos aspectos da saúde, tanto na infância quanto na fase adulta. A partir da idade escolar, as crianças começam a exercer autonomia crescente na decisão de quando querem e o que querem se alimentar ${ }^{1}$. Essa autonomia, se não for estimulada em um ambiente saudável, pode ser um dos fatores responsáveis pelo aumento considerável dos casos de obesidade infantil ${ }^{8}$.
Ainda em relação à obesidade frente às políticas de saúde, trazemos que os custos acarretados pelo Sistema Único de Saúde (SUS) - devido à expansão dessa epidemia no século XXI - são estimados para um ano com todas as doenças relacionadas ao sobrepeso e à obesidade: o custo do câncer, diabetes e doenças cardiológicas é de cerca de 20 bilhóes de dólares; as hospitalizaçóes custam, aproximadamente, 1,5 bilhão; e os procedimentos de ambulatoriais, por volta de 680 milhōes de dólares?.

Assim, os custos com a obesidade têm se tornado preocupantes nos últimos anos, visto que apresentam impacto financeiro no sistema de saúde (custos diretos), e pela perda de produtividade e qualidade de vida (custos indiretos) dessas pessoas. Portanto, a obesidade se mostra como um desafio da saúde para os profissionais e todos os setores sociais9. Partindo desse pressuposto, tem-se como objeto de estudo "o excesso de peso na criança". Assim, nosso objetivo é identificar e revisar como as publicaçóes científicas discutem os fatores que influenciam o aumento de peso nas crianças, e assim, propor possíveis intervençóes de enfrentamento.

\section{Metodologia}

Revisar, sistematicamente, artigos que consideraram a associação entre comportamento alimentar, estado nutricional e obesidade em crianças. A pesquisa dos artigos foi feita nas bases de dados Medline/PubMed e Scientific Eletronic Library Online (SciELO-Brasil), na qual a busca foi conduzida em janeiro de 2014. Os termos de busca utilizados na Medline/PubMed foram: "obesity children" combinado com "feeding behavior". No SciELO-Brasil, as palavras-chaves utilizadas foram: "obesidade infantil" e "escolares".

Estabeleceram-se os seguintes critérios de inclusão: artigos de livre acesso e disponíveis na íntegra para download; em língua vernácula e inglesa; pesquisas com ambos os sexos; crianças de 0 a 12 anos, seguindo as publicaçōes de janeiro de 2009 a dezembro de 2013.

Deliberou-se náo considerar artigos que apresentassem dados alusivos a: população adulta; artigos não disponíveis na íntegra; notas científicas; amostras representativas de população indígena; dados de apenas um dos sexos; amostra contendo sujeitos portadores de outras enfermidades; além da obesidade e artigos de relato de experiência.

Na busca realizada no PubMed, encontrou-se o total de 3.257 artigos, dos quais, após aplicação dos filtros "texto completo", "últimos cinco anos" e "seres humanos", restaram 485. Destes, 104 foram selecionados a partir dos títulos, restando 15 que se encaixavam aos 
critérios de inclusão e exclusão preestabelecidos. No levantamento realizado na SciELO-Brasil, foram encontrados 104 artigos, com aplicação dos idiomas restaram 98, utilizando o corte temporal restaram 48. Destes, retiramos 11 artigos que não pertenciam a estudos que abrangiam o objetivo do trabalho. Assim, foram analisadas 30 publicações da SciELO. Salienta-se a inclusão de referências dos artigos lidos, e ao final foram selecionados 54 artigos que discutiam o comportamento alimentar, estado nutricional e obesidade em crianças, sendo que a expressão "excesso de peso" será usada no artigo em referência tanto ao diagnóstico de sobrepeso como de obesidade, para fins de uniformização de terminologia e mais fácil compreensão (Tabela 1 ).

Os dados foram analisados a partir da leitura dos resumos e da utilizaçáo dos dados apresentados. Para a categorização dos estudos, utilizou-se a análise de conteúdo, em que as etapas se constituem de pré-análise, exploraçáo do material e tratamento dos resultados ${ }^{10}$.

Tabela 1: Detalhamento do percurso metodológico e desfecho dos artigos selecionados.

\begin{tabular}{lc}
\hline Base de dados pesquisada & $\begin{array}{c}\text { Número de artigos } \\
\text { encontrados }\end{array}$ \\
\hline PubMed (unitermos e filtros) & 3.257 \\
Obesity children + feeding behavior & 793 \\
Texto completo & 485 \\
2009 a 2013 + estudos com seres & \\
humanos & \\
SciELO (palavra-chave + filtros) & 104 \\
Obesidade infantil & 98 \\
Português e inglês & 48 \\
2009 a 2013 & 37 \\
Estudos náo condizentes com o objetivo & \\
Critérios de corte & 143 \\
Artigos com populaçóes adultas ou & \\
pré-escolares & 212 \\
Artigos com duplicidades & 52 \\
Notas científicas & 63 \\
Artigos incluindo populaçáo indígena & 44 \\
Artigos com apenas um dos sexos & 31 \\
Artigos relacionando anemia com & \\
doenças (cardiovasculares etc.) & \\
Total & 545 \\
Artigos incluídos & \\
Pubmed & \\
SciELO & \\
Total de artigos analisados & \\
\hline
\end{tabular}

Fonte: Recursos próprios de pesquisa.

\section{Resultados e discussões}

Os artigos analisados permitiram o apontamento da situação do excesso de peso na população infantil no Brasil e em alguns países desenvolvidos, num período de cinco anos. Eles contribuem para a verificação das reais condiçóes em que essa epidemia do século XXI vem sendo disseminada, como afeta a saúde das crianças e como onera os serviços de saúde.

\section{Caracterização das publicações científicas no Brasil e no mundo, relacionadas ao excesso de peso na infância}

Houve maior número de pesquisas realizadas sobre a temática entre os anos de 2011 e 2012 . Tal assertiva demonstra que o interesse pelo assunto e a necessidade de respostas aumentaram gradativamente, e que estudos transversais e de intervenção são necessários.

Dos artigos analisados, foram três em Portugal dois na Inglaterra, dois na Austrália, um na Argentina, um na Malásia e um na Alemanha. A produção brasileira sobre a obesidade infantil apresentou-se da seguinte maneira: um na regiâo Norte, seis no Nordeste, seis no Sul, treze no Sudeste.

Em relação ao desenho dos estudos: 21 são de corte transversal, 16 de revisão de literatura, dois são retrospectivos, um é do tipo epidemiológico e um longitudinal. A faixa etária dos participantes dos estudos variou de menos de um ano a quinze anos de idade. Em relação ao tamanho amostral, obtiveram-se estudos representativos, com amostra de 15.144 crianças, realizado em diversos países ${ }^{11}$.

Um roteiro de análise foi construído para organização e exposição dos resultados, destacando-se: os autores da pesquisa; ano de publicação; ano de estudo; regiăo geográfica brasileira/país; tipo de estudo; idade da criança e tamanho amostral (Tabela 2).

A partir das variáveis investigadas em cada artigo, os estudos foram agrupados em três categorias: a) obesidade infantil e serviço de saúde (15 artigos); b) Obesidade infantil e hábitos familiares (6 artigos); c) Obesidade infantil e ambiente escolar (23 artigos). Neles, foram analisados os fatores que influenciaram o aumento de peso na populaçáo infantil, assim como possíveis intervençôes para tentar minimizar esse problema de saúde pública (Tabela 3). 
Tabela 2: Publicações sobre obesidade infantil no Brasil e no mundo; dados de estudos entre os anos de 2009 e 2013.

\begin{tabular}{|c|c|c|c|c|c|c|}
\hline Autores & $\begin{array}{c}\text { Ano de } \\
\text { publicaçáa }\end{array}$ & Ano de estudo & $\begin{array}{c}\text { Regiáo } \\
\text { brasileira/país }\end{array}$ & Tipo de estudo & Idade da criança & $\begin{array}{c}\text { Tamanho } \\
\text { amostral /No de } \\
\text { estudos }\end{array}$ \\
\hline Santos et al. & 2011 & 2008 & Nordeste & Transversal & 7 a 10 anos & 8 sujeitos \\
\hline Azevedo et al. & 2012 & $2010 / 2011$ & Sudeste & Revisão & N. A. & 51 artigos \\
\hline Moraes et al. & 2013 & N. I. & Nordeste & Transversal & N. A. & 7 sujeitos \\
\hline Pergher et al. & 2010 & 2009 & Sudeste & $\begin{array}{l}\text { Revisão de } \\
\text { literatura }\end{array}$ & N. A. & 54 artigos \\
\hline Pereira et al. & 2013 & 2012 & Sudeste & Revisão & N. A. & 28 artigos \\
\hline Venâncio & 2012 & $2009 / 2010$ & Portugal & Transversal & 6-12 anos & 623 sujeitos \\
\hline Leal et al. & 2012 & 2006 & Nordeste & Transversal & $\begin{array}{l}\text { 5-9 anos } \\
5-19 \text { anos }\end{array}$ & $\begin{array}{l}700 \text { sujeitos } \\
1.435 \text { sujeitos }\end{array}$ \\
\hline Lindsay et al. & 2012 & 2006 & Argentina & Revisão & N. A. & 38 artigos \\
\hline Tenório et al. & 2011 & 2010 & Sudeste & Revisão & N. A. & 38 artigos \\
\hline Carvalho et al. & 2011 & 2010 & Portugal & Revisão & N. A. & 53 artigos \\
\hline Rodrigues et al. & 2010 & N. I. & Portugal & Revisão & N. A. & 72 artigos \\
\hline $\begin{array}{l}\text { Marchi- Alves } \\
\text { et al. }\end{array}$ & 2011 & $2008 / 2009$ & Sudeste & Retrospectivo & N. A. & $\begin{array}{c}193 \text { prontuários e } \\
18 \text { artigos }\end{array}$ \\
\hline Marins et al. & 2011 & 2004 & Sudeste & Transversal & 3-6 anos & 369 \\
\hline Friedrich et al. & 2012 & 2011 & Sul & $\begin{array}{l}\text { Revisão } \\
\text { sistemática }\end{array}$ & N. A. & 55 artigos \\
\hline Lourenço et al. & 2009 & 2008 & Sudeste & Revisão & N. A. & 33 artigos \\
\hline Rossi et al. & 2010 & 2008 & Sul & Revisão & N. A. & 46 artigos \\
\hline Novaes et al. & 2009 & 2005 & Sudeste & Transversal & $6-10$ anos & 769 sujeitos \\
\hline Mendoça et al. & 2010 & 2009 & Nordeste & Epidemiológico & 7-13 anos & 909 sujeitos \\
\hline Quadros et al. & 2012 & $2004 / 2005$ & Sul & N. I. & 6-9 anos & 585 sujeitos \\
\hline $\begin{array}{l}\text { Rodrigues L.G } \\
\text { et al. }\end{array}$ & 2011 & 2010 & Sudeste & Revisão & N. A. & 56 artigos \\
\hline Reis et al. & 2011 & 2009 & Sudeste & Revisão 1 & N. A. & 49 artigos \\
\hline Damasceno et al. & 2009 & 2008 & Sudeste & Transversal & $6-24$ meses & 95 sujeitos \\
\hline Corso et al. & 2012 & 2008 & Sul & Transversal & 6-10 anos & 4.964 sujeitos \\
\hline Rech et al. & 2010 & 2009 & Sul & Transversal & $7-12$ anos & 1.442 sujeitos \\
\hline Krinski et al. & 2011 & 2008 & Norte & Transversal & $\begin{array}{l}\text { 6-17 anos } \\
\text { 6-11 anos }\end{array}$ & $\begin{array}{c}5.883 \\
2.765 \text { sujeitos }\end{array}$ \\
\hline Moreira et al. & 2012 & 2007 & Nordeste & Transversal & Menor de 5 anos & 963 sujeitos \\
\hline Momoi et al. & 2012 & 2006 & Sudeste & Retrospectiva & Menor de 1 ano & 414 sujeitos \\
\hline Damasceno et al. & 2010 & 2008 & Nordeste & Transversal & 6-11 anos & 727 sujeitos \\
\hline Gilman et al. & 2013 & 2013 & Inglaterra & Revisão & N. A. & 8 artigos \\
\hline Naidu et al. & 2013 & 2006 & Malásia & Base populacional & 7-12 anos & 7.749 sujeitos \\
\hline $\begin{array}{l}\text { Marion Hare } \\
\text { et al. }\end{array}$ & 2012 & N. I. & N. I. & N. I. & 4-7 anos & 270 sujeitos \\
\hline
\end{tabular}

Legenda: N. A. - Não apropriado; N. I. - Não indica.

Fonte: Recursos próprios de pesquisa. 2014. 
Tabela 3: Fatores que influenciam o aumento de peso nas crianças segundo os serviços de saúde e possíveis intervenções.

\begin{tabular}{|c|c|}
\hline \multirow{4}{*}{ 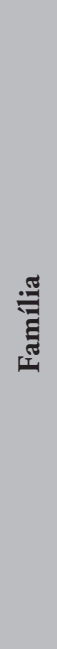 } & Fatores que influenciam o aumento de peso \\
\hline & $\begin{array}{l}\text { A ausência da figura paterna pode determinar a gênese e manutenção da obesidade infantil. } \\
\text { Deficiência na atividade física dos pais e, consequentemente, dos filhos. } \\
\text { Ausência de apoio para mudança de hábitos. } \\
\text { Baixo consumo de frutas e produtos hortícolas. } \\
\text { Desmame precoce. } \\
\text { Estado nutricional de pais e mães. } \\
\text { Permissividade alimentar. } \\
\text { Excesso de uso da televisão ou de jogos eletrônicos. } \\
\text { Estigma social e não aceitação social da obesidade infantil. } \\
\text { Baixa autoestima. } \\
\text { Desestruturação familiar. }\end{array}$ \\
\hline & Possíveis intervençóes \\
\hline & $\begin{array}{l}\text { Ações de prevenção do ganho excessivo de peso na infância: base comunitária. } \\
\text { Açóes de prevenção: base comportamental. }\end{array}$ \\
\hline \multirow{4}{*}{ 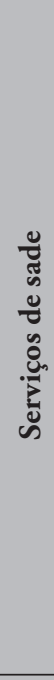 } & Fatores influentes no aumento de peso \\
\hline & $\begin{array}{l}\text { A duração do sono curto associado à diminuição dos níveis de leptina } \\
\text { Diminuição de e consequente aumento dos níveis de grelina } \\
\text { Desconhecimento da doença e seu tratamento } \\
\text { Comportamentos preconceituosos } \\
\text { Políticas públicas verticalizadas }\end{array}$ \\
\hline & Possíveis Intervençôes \\
\hline & $\begin{array}{l}\text { Esclarecimento sobre a patologia aos pais e à criança } \\
\text { Apoio familiar } \\
\text { Diagnóstico precoce } \\
\text { Educaçáo Popular em Saúde } \\
\text { Tratamento Supervisionado por uma equipe de saúde } \\
\text { Conselhos de familiares sobre a procura do serviço } \\
\text { Participação ativa dos pais no tratamento } \\
\text { Segmentação da duração do sono }\end{array}$ \\
\hline \multirow{4}{*}{ 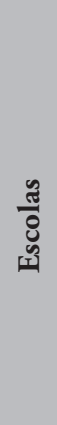 } & Fatores Influentes no aumento de peso \\
\hline & $\begin{array}{l}\text { Não tomar café da manhã. } \\
\text { Realizar menos de três refeições por dia. } \\
\text { Publicidade de alimentos para crianças. }\end{array}$ \\
\hline & Possíveis intervençóes \\
\hline & $\begin{array}{l}\text { Realizar conscientização da importância da alimentação. } \\
\text { Criação de políticas públicas horizontais. } \\
\text { Realizar trabalho intersetorial e multiprofissional. }\end{array}$ \\
\hline
\end{tabular}

Fonte: análise dos artigos selecionados para o estudo. 


\section{Estruturação dos artigos segundo análise dos subgrupos das publicações}

\section{Obesidade infantil e situação familiar}

A família apresenta-se como um sistema mesclado por diversos integrantes, ou subsistemas, que interatuam e se influenciam mutuamente. Assim, é compreensível que a obesidade em um dos membros ocasione mudanças no sistema familiar. Os hábitos familiares influenciam diretamente o estado nutricional e o comportamento alimentar das crianças. Um estudo relata que a instabilidade conjugal pode ser um dos motivos para desenvolver o excesso de peso na infância, progredindo na adolescência até a vida adulta, visto que se trata de uma doença complexa e crônica ${ }^{12,34,44}$.

Assim, quando casais estão separados ou em um ambiente hostil, exibem uma tentativa de aproximaçáo com o filho, por meio de um diálogo representado pela permissividade alimentar. No entanto, a figura paterna pode representar a gênese e a manutençáo da obesidade, principalmente porque sua presença pode reorganizar o ambiente familiar, favorecendo o manejo da obesidade infantil ${ }^{13}$.

$\mathrm{O}$ excesso de peso e a obesidade têm sido encontrados com frequência a partir dos cinco anos de idade, em todos os grupos socioeconômios e regióes brasileiras ${ }^{1,2,5,6,14}$.

A família é responsável pela formação do comportamento alimentar da criança, por meio de uma aprendizagem sociocultural, sendo os pais os primeiros educadores nutricionais. Dessa maneira, como outros ambientes socialmente influentes, o núcleo familiar interage com as predisposições genéticas e psicológicas à obesidade infantil ${ }^{15,29}$.

Entendendo que a genética é um fator crucial no risco do excesso de peso, avaliou-se que as crianças cujas mães eram obesas apresentavam sete vezes mais chance de sobrepeso, em comparação àquelas que não tinham mães obesas. Da mesma forma, crianças que eram unigênitas passavam, por dia, três horas ou mais em frente à televisão, ou não praticavam educação física na escola, apresentando de duas a cinco vezes mais chances de desenvolver sobrepeso, em relação àquelas que não apresentaram essas características ${ }^{16,31}$.

Importante salientar que, ao se tratar de excesso de peso em crianças, há um estigma que necessita ser rompido, pois algumas famílias ainda se apoiam no simbolismo saúde-gordura, que acaba por dificultar o emagrecimento, reforçando o aspecto cultural de que a criança sadia é a que nasce "gordinha e bonita". É notória a dificuldade por parte dos familiares em perceber a obesidade na criança ${ }^{12,30}$.

Um estudo realizado com mães de baixa renda em Buenos Aires, Argentina, descobriu que muitas mães estavam cientes de que a obesidade pode ser prejudicial à saúde da criança, mas a maioria delas não sabe sobre suas consequências reais, nem que o trabalho materno, pressóes familiares, insegurança alimentar e preocupações financeiras parecem influenciar a prática inadequada de alimentação infantil. É uma realidade que se assemelha à brasileira ${ }^{17}$.

Estudos corroboram que, embora os fatores genéticos predisponham o desenvolvimento da obesidade, os principais determinantes de seu aumento parecem ser os fatores ambientais e comportamentais, como a falta de atividade física, maior tempo assistindo à televisão, balanço energético negativo e aumento do consumo de alimentos ricos em açúcares e gorduras ${ }^{18}$.

\section{Obesidade infantil e serviço de saúde}

A obesidade infantil está frequentemente associada a outras doenças crônicas, como as cardiovasculares, destacando-se a hipertensão, dislipidemia, intolerância à glicose, apneia do sono e infertilidade, que contribuem para um risco acrescido de morte prematura e perda de qualidade de vida ${ }^{19,33}$.

Com a finalidade de obter um diagnóstico precoce e prevenir o ganho de peso na infância, algumas medidas simples podem ser adotadas em consultas médicas e até em avaliaçóes escolares periódicas. A avaliação de obesidade abdominal é uma importante medida, por seu impacto na saúde cardiovascular. Embora ainda não haja um consenso ou padronização de valores de circunferência abdominal para essa população jovem, diversos estudos já mostram aumento na prevalência dessa condição em crianças e adolescentes ${ }^{1,39}$.

Para um possível diagnóstico, estudos no Brasil e na Alemanha investigaram a associação entre índice de massa corporal (IMC), circunferência da cintura (CC), cintura-altura e IMC em crianças. Tais estudos chegaram à conclusão que o uso isolado da CC ou do IMC é ineficaz na determinação do padrão de gordura na infância, observando-se que, na prática da enfermagem, sugere-se a utilização de ambas as medidas antropométricas estudadas, para a determinação da distribuição de gordura em crianças. Como meio de triagem para a hipertensão arterial, sugere-se a resistência insulínica e as dislipidemias em crianças e adolescentes, tanto nas unidades básicas de saúde, quanto nas escolas ou serviços especializados ${ }^{20,36}$. Avalia-se que quanto maior a cintura, mais riscos a criança possui para desenvolver problemas de saúde ${ }^{21,38}$.

Estudos realizados em Portugal ${ }^{19}$ retratam, em conjunto, a problemática da obesidade infantil, delineando os métodos de abordagem, analisando qual seria mais resolutivo: o método de base comportamental ou o de base comunitária, chegando à conclusão de que as intervenções em ambiente familiar de base comportamental 
que incorporam modificações ao nível da alimentação e da atividade física parecem ser as medidas mais efetivas no controle do peso corporal. No entanto, as intervençôes de base comunitária, apesar de ainda serem escassas, parecem assumir um eixo estratégico no combate à doença. A associaçáo feita entre o marketing de produtos alimentares e sua elevada densidade energética, atrelados à obesidade infantil, demonstra que a maior parte dos alimentos publicitários dirigidos às crianças sáo ricos em calorias, gorduras, açúcar e sal. A grande questão é que as crianças representam um alvo bastante vulnerável, pela sua incapacidade de percepção das intençóes persuasivas da publicidade ${ }^{22}$. Assim, tem-se que a mídia televisiva engana as crianças e alienam os pais.

Porém, a revisão realizada pelo pesquisador alega que a compreensão da publicidade televisiva pelas crianças parece depender de alguns fatores, sendo a idade um dos mais determinantes. De forma geral, antes dos quatro ou cinco anos de idade, as crianças veem a publicidade apenas como informativa, e náo a distinguem facilmente da programaçáo. Entre os quatro e os sete anos, ficam aptas para distinguir publicidade de programação, e apenas aos oito anos percebem a intenção de persuasão. Só após os onze ou doze anos podem construir um pensamento crítico acerca da publicidade e das intençóes dos publicitários. Conclui-se que, à medida que a idade avança, o criticismo das crianças aumenta em relação à informação fornecida pela publicidade, bem como sua intenção persuasiva ${ }^{22}$.

Um estudo transversal que teve amostra de 1.856 crianças de três a cinco anos em Toronto avaliou a possível associação entre níveis séricos de colesterol e comportamentos alimentares durante a infância. Com ele, chegou-se ao resultado de que não existe necessariamente níveis alterados nas crianças quando apresentam comportamentos alimentares inadequados, mas sim que esses níveis se encontram em certo limiar, o que caracteriza risco para doenças cardiovasculares futuras. Portanto, esse é um dos fatores que os serviços de saúde podem trabalhar para prevenir e controlar o excesso de peso na infância ${ }^{23}$.

\section{Obesidade infantil e escola}

É notório que o ambiente escolar tem grande influência na saúde da criança, seja direta ou indiretamente. Isso se deve não só pelo tempo de permanência, mas também como local de convívio e troca de informaçóes sobre saúde. Visto todas essas razóes, considera-se a escola como o ambiente ideal para implementação de estratégias de prevenção e controle de questóes de saúde, como a obesidade ${ }^{1,46}$.

Um estudo epidemiológico realizado em uma capital brasileira informa que a prevalência encontrada nessa população foi menor do que os resultados de estudos com populaçóes similares. Apesar desses resultados, os autores conseguiram estimar que o risco de obesidade é cinco vezes maior em escolas particulares. A obesidade foi mais frequente situa-se na faixa etária de sete a nove anos, revelando a necessidade de monitorizaçáo desses indivíduos, em longo prazo, para evitar futuras complicações $^{24}$.

Um estudo transversal realizado com 623 escolares de Portugal constatou a prevalência de sobrepeso/obesidade em $28,4 \%$ deles, sendo $15,7 \%$ com sobrepeso e $12,7 \%$ com obesidade. Quanto à distribuição por gênero e idade, não obteve-se diferença em relação aos sexos. No entanto, na faixa dos seis anos há mais meninas obesas, e na faixa dos nove, há maior concentraçáo de obesidade em meninos ${ }^{25}$.

Enquanto isso, um estudo realizado com escolares brasileiros, de 7 a 12 anos, demonstrou uma prevalência menor, com $8 \%$ para obesidade e $19,9 \%$ para sobrepeso ${ }^{26}$.

O comportamento alimentar das crianças é influenciado pelos hábitos da família e pelo seu convívio social. Desse modo, é importante observar que as crianças consomem os alimentos mais próximos e de mais fácil acesso. Diante desse pressuposto, um estudo longitudinal realizado em Londres, na Inglaterra, procurou investigar se os ambientes ao redor das escolas afetariam a dieta. Observou-se, em quatro anos de investigação, que existe influência na dieta dos escolares, e que geralmente alimentos não saudáveis são consumidos, como batata frita, salgados e refrigerante ${ }^{27}$. Isso indica que, ao desenvolver intervençóes nos escolares, o conhecimento da territorialidade da escola, além do conhecimento da dinâmica da família e do sujeito é necessário para que essas medidas sejam efetivas e resolutivas.

Destaca-se ainda que a criança que toma café da manhá tem 21,3\% menos chances de apresentar excesso de peso. Isso indica que o número de refeiçôes diárias apresenta associaçáo inversa com obesidade e sobrepeso, ou seja, os escolares que realizam três refeiçóes ou menos por dia apresentaram $48 \%$ mais chances para obesidade e sobrepeso ${ }^{26}$.

Dois estudos internacionais, um realizado nos Estados Unidos e outro na Austrália, evidenciam que a duração do sono é um fator que pode desencadear risco de desenvolver excesso de peso na criança, especialmente entre os grupos etários mais jovens ${ }^{27}$, acarretando também no prejuízo do tratamento contra a obesidade ${ }^{28}$.

\section{Conclusão}

Diante do presente estudo, observa-se que a escola, o serviço de saúde e a família são a tríade basilar do diagnóstico, tratamento e prevenção da obesidade infantil, 
visto que se trata de uma doença complexa, crônica, e que exige um esforço mútuo para que se possa buscar a solução nas intervençóes de enfrentamento desse grave problema da saúde pública.

Observa-se também que há uma associação clara entre a obesidade dos pais e a dos filhos, Não obstante, o mesmo nível de evidência náo existe para outras variáveis, tais como o sexo da criança, o peso ao nascer, o tempo de aleitamento materno e a escolaridade materna.

Destarte, deve-se salientar a ausência de consenso relativo à classificação de obesidade em crianças e adolescentes, ao contrário dos adultos. No entanto, se faz consenso que as crianças com excesso de peso têm risco acrescido de se tornarem adultos obesos, e que se gasta muito com as consequências advindas dessa patologia.

Portanto, a fim de buscar caminhos para implementar políticas públicas horizontais que visam a saúde das crianças, salientamos algumas intervençóes intersetoriais, por vezes até medidas simplórias, mas que, se construídas visualizando o sujeito, e não apenas sua patologia, tornam-se essenciais, tais como: intervençóes de duração superior a um ano, com avaliaçóes periódicas; realização da Educação Popular em Saúde; e introduçáo dessa temática nas disciplinas da escola. Não obstante, também deve-se incentivar o envolvimento dos pais nessa discussão, ingressar a educação nutricional no ensino de forma interdisciplinar, e fornecer, por meio da escola, uma alimentação saudável às crianças, assim como desenvolver estratégias que aumentem a prática de atividades físicas de lazer.

Partindo desse pressuposto, deixamos nossa contribuição acerca da discussão que permeia a obesidade infantil no Brasil e no mundo, almejando que novas publicaçóes possam surgir.

\section{Referências}

1. Azevedo FR, Brito B. Influência das variáveis nutricionais e da obesidade sobre a saúde e o metabolismo. Rev Assoc Med Bras. abr 2012;58(6);714-723. Disponível em http://www.scielo.br/ pdf/ramb/v58n6/v58n6a18.pdf [Acesso em 3 jan 2014].

2. Ferreira VA, Magalhães R. Obesidade no Brasil: tendências atuais. Revista portuguesa de saúde pública. Jul/dez 2006;24(2);71-82. Disponível em http://www.ensp.unl. pt/dispositivos-de-apoio/cdi/cdi/sector-de-publicacoes/ revista/2000-2008/pdfs/2-06-2006.pdf [Acesso em 10 jul. 2013].

3. World Health Organization. Forum and Technical Meeting on Population-based Prevention Strategies for Childhood Obesity. Geneva: WHO; 2009.

4. Pesquisa de Orçamentos Familiares - 2008-2009: antropometria e estado nutricional de crianças, adolescentes e adultos no Brasil. Rio de Janeiro. Instituto Brasileiro de Geografia e Estatística (IBGE). 2010. Disponível em http:// www.ibge.gov.br/home/. [Acesso em 15 set 2015].

5. Oliveira CL, Fisberg M. Obesidade na infância e adolescência: uma verdadeira epidemia. Arq Bras Endocrinol Metab. 2003;47(2). Disponível em http://www.scielo.br/ pdf/abem/v47n2/a01v47n2.pdf. [Acesso em 3 ago. 2013].

6. Andrade, DEG. Prevalência de sobrepeso e obesidade em crianças de escolas públicas e privadas do ensino fundamental da cidade de Franca-SP e alguns fatores de risco associados. [dissertação de mestrado em Saúde na Comunidade] Ribeirão Preto: Faculdade de Medicina de Ribeirão Preto, Universidade de São Paulo, 2006. Disponível em http://www.teses.usp.br/teses/disponiveis/17/17139/tde18092006-162308/pt-br.php. [Acesso em 6 ago. 2013].

7. IOTF, International Obesity Taskforce. A prevalência de obesidade em todo o mundo. Londres. Disponível em http://www.worldobesity.org. [Acesso em 31 ago. 2013].

8. Ministério da Saúde. Secretaria de Atenção à Saúde. Departamento de Atenção Básica. Cadernos de Atenção Básica: Obesidade. Brasília (DF). 2006. Disponível em http://www.prosaude.org/publicacoes/diversos/cad_AB_ obesidade.pdf. [Acesso em 10 ago. 2013].

9. Custos de Doenças Ligadas à Obesidade para o SUS. Higienópolis: Abeso. 2012. Disponível em http://www.abeso.org.br/lenoticia/909/custos+de+doencas+ligadas+a+obesidade+para+o+sus.shtml . [Acesso em 5 jan 2014].

10. Bardin L. Análise de Conteúdo. Lisboa. Ediçôes 70;2000.

11. Lissner L. et al. Television habits in relation to overweight, diet and taste preferences in European children: the IDEFICS study. Eur J Epidemiol. Set 2012;27(9):70515. Disponível em: http://www.ncbi.nlm.nih.gov/pub$\mathrm{med} / 22911022$. [Acesso em 15 set 2015].

12. Moraes PM, Dias CMDSB. Nem só de pão se vive: a voz das mães na obesidade infantil. Psicol. cienc. Prof. 2013;33(1):4659. Disponível em: http://www.scielo.br/scielo.php?scrip$\mathrm{t}=$ sci_arttext\&pid=S1414-98932013000100005\&lang=pt. [Acesso em 15 set 2015].

13. Santos LRDC, Rabinovich EP. Situaçóes familiares na obesidade exógena infantil do filho único. Saude soc. 2011;20(2):50721. Disponível em: http://www.scielo.br/scielo.php?scrip$\mathrm{t}=\mathrm{sci} \_$arttext\&pid=S0104-12902011000200021\&lang=pt. [Acesso em 15 set 2015].

14. Simon VGN, et al. Prevalência de sobrepeso e obesidade em crianças de dois a seis anos matriculadas em escolas particulares no município de São Paulo. Revista Brasileira de Crescimento e Desenvolvimento Humano. 2009;19(2). Disponível em: http://pepsic.bvsalud.org/scielo.php?scrip$\mathrm{t}=$ sci_arttext\&pid=S0104-12822009000200002. . [Acesso em 15 set 2015 ].

15. Murdoch M, et al. Family-based behavioural management of childhood obesity: Service evaluation of a group programme run in a community setting in the United Kingdom. European Journal of Clinical Nutrition, 2011;65:764-7. 
16. Novaes JF. Fatores ambientais associados ao sobrepeso infantil. Rev. Nutr., Campinas, set/out 2009;22(5):66173. Disponível em http://www.scielo.br/pdf/rn/v22n5/ v22n5a07.pdf . [Acesso em 3 jan. 2014].

17. Lindsay AC et al. Child feeding practices and household food insecurity among low-income mothers in Buenos Aires, Argentina. Ciênc. saúde coletiva.2012;17(3):661-9. Disponível em: http://www.scielo.br/scielo.php?script=sci_arttext\&pid=S1413-81232012000300012\&lang=pt. [Acesso em 15 set 2015].

18. Tenorio AES, Cobayashi F. Obesidade infantil na percepção dos pais. Rev. paul. pediatr. 2011;29(4):634-9. Disponível em: http://www.scielo.br/scielo.php?script=sci_arttext\&pi$\mathrm{d}=$ S010305822011000400025\&lang=pt. [Acesso em 15 set 2015].

19. Carvalho MA, Carmob I, Bredac J, Rito AI. Análise comparativa de métodos de abordagem da obesidade Infantil. Rev Port Saúde Pública. 2011;29(2):148-56. Disponível em http://www.scielo.gpeari.mctes.pt/pdf/rpsp/v29n2/ v29n2a08.pdf . [Acesso em 4 jan 2015].

20. Damasceno MMC et al. Correlação entre índice de massa corporal e circunferência da cintura em crianças. Acta Paul Enferm. 2010;23(5);652-67. Disponível em http://www. scielo.br/pdf/ape/v23n5/11.pdf . [Acesso em 4 jan 2015].

21. Kesztyüs D et al. Is central obesity associated with poorer health and health-related quality of life in primary school children? Cross-sectional results from the Baden-Württemberg Study. BMC Public Health. 2013;13:260. Disponível em: http://www.ncbi.nlm.nih.gov/pmc/articles/PMC3652747. [Acesso em 15 set 2015].

22. Rodrigues LG, Pombo N, Koifman S. Prevalência de alteraçóes metabólicas em crianças e adolescentes com sobrepeso e obesidade: uma revisão sistemática. Rev Paul Pediatr. 2011 [acesso em 16 set 2015];29(2):277-88. Disponível em http://www.scielo.br/pdf/rpp/v29n2/a21v29n2.pdf. [Acesso em 4 jan 2014].

23. Persaud $\mathrm{N}$ et al. Association between serum cholesterol and eating behaviours during early childhood: a cross-sectional study. CAMJ. 2013;185(11):536-42. Disponível em: http://www.ncbi.nlm.nih.gov/pmc/articles/PMC3735770. [Acesso em 15 set 2015].

24. Mendonça MRT et al. prevalência de sobrepeso e obesidade em crianças e adolescentes da cidade de Maceió. Rev Assoc Med Bras. 2010;56(2):192-6. Disponível em: http://www.scielo.br/ pdf/ramb/v56n2/a18v56n2.pdf. [Acesso em 3 jan 2014].

25. Venâncio P, Aguilar S, Pinto G. Obesidade infantil: um problema cada vez mais actual Obesity in children in Portugal: a cross-sectional study. Rev Port Med Geral Fam. 2012;28(6);410-6. Disponível em: http://www.scielo.gpeari.mctes.pt/scielo.php?script=sci_arttext\&pid=S2182=51732012000600005-\&langpt. [Acesso em 15 set 2015].

26. Rech, RR et al. Prevalência de obesidade em escolares de 7 a 12 anos de uma cidade serrana do RS, Brasil. Rev Bras Cineantropom Desempenho Hum. 2010;12(2): 90-7.
Disponível em :http://www.scielo.br/pdf/rbcdh/v12n2/ a02v12n2.pdf. [Acesso em 3 jan 2014].

27. Smith DSC et al. Does the local food environment around schools affect diet? Longitudinal associations in adolescents attending secondary schools in East London. BMC Public Health. 2013;13(70). Disponível em: http://www.ncbi.nlm. nih.gov/pmc/articles/PMC3567930. [Acesso em 15 set 2015].

28. Lisa M et al. The Association Between Sleep Duration and Weight in Treatment-Seeking Preschoolers with Obesity. Sleep Med. 2012;13(8):1102-5. Disponível em: http:// www.ncbi.nlm.nih.gov/pmc/articles/PMC3433160. [Acesso em 15 set 2015].

29. Rossi CE et al. Influência da televisão no consumo alimentar e na obesidade em crianças e adolescentes: uma revisão sistemática. Rev. Nutr. Campinas. 2010;23(4): 60720. Disponível em http://www.scielo.br/pdf/rn/v23n4/ v23n4a11.pdf. [Acesso em 3 jan 2014].

30. Moreira MA et al. Overweight and associated factors in children from northeasten Brazil. J Pediatr Rio J. 2012;88(4):347-52. Disponível em http://www.scielo.br/ pdf/jped/v88n4/a12v88n4.pdf. [Acesso em 3 jan 2014].

31. Alexandra $\mathrm{C}$ et al. An analysis of potential barriers and enablers to regulating the television marketing of unhealthy foods to children at the state government level in Australia. BMC Public Health. 2012;13(1123). Disponível em http://www.ncbi.nlm.nih.gov/pmc/articles/PMC3572413. [Acesso em 15 set 2015].

32. Bernardo $\mathrm{CO}$ et al. Factors associated with nutritional status of 7-10 year-old schoolchildren: sociodemographic variables, dietary and parental nutritional status. Rev Bras Epidemiol. 2012;15(3):651-61. Disponível em http://www.scielosp.org/ pdf/csp/v29n2/03.pdf. [Acesso em 3 jan 2014].

33. Shi $Z$ et al. Short sleep duration and obesity among Australian children. BMC Public Health. 2010;10(609). Disponível em: http://www.ncbi.nlm.nih.gov/pmc/articles/ PMC2964630. [Acesso em 15 set 2015].

34. Claudia $S$ et al. The stigma of obesity in the general public and its implications for public health - a systematic review. BMC Public Health. 2011;11(661). Disponível em: http://www.ncbi.nlm.nih.gov/pmc/articles/PMC3175190. [Acesso em 15 set 2015].

35. Lourenço BH, Cardoso MA. Práticas alimentares na infância, crescimento infantil e obesidade na vida adulta. Arq Bras Endocrinol Metab. 2009;5: 528-39. Disponível em: http://www.scielo.br/scielo.php?script=sci_arttext\&pid=S0004-7302009000500006\&lang=pt. [Acesso em 15 set 2015 ].

36. Quadros TMB et al. Excesso de peso em crianças: comparação entre o critério internacional e nacional de classificação do índice de massa corpórea. Rev Paul Pediatr. 2012;3(4):537-43. Disponível em: http://www.scielo.br/ pdf/rpp/v30n4/12.pdf. [Acesso em 3 jan 2014].

37. Rajalakshmi L et al. Protocol for systematic reviews of determinants/correlates of obesity-related dietary and physical 
activity behaviors in young children (preschool 0 to 6 years): evidence mapping and syntheses. Syst Rev. 2013;2(28). Disponível em: http://www.ncbi.nlm.nih.gov/pmc/articles/ PMC3691606. [Acesso em 15 set 2015].

38. Friedrich RR, Schuch II, Wagner MB. Efeito de intervençóes sobre o índice de massa corporal em escolares. Rev Saúde Pública. 2012;46(3):551-60. Disponível em: http://www. scielo.br/pdf/rsp/v46n3/3678.pdf . [Acesso em 3 jan 2014]

39. Marchi-Alves LM et al. Obesidade infantil ontem e hoje: importância da avaliaçáo antropométrica pelo enfermeiro. Esc Anna Nery. 2011 [acesso em 16 set 2015];15(2): 23844. Disponível em: http://www.scielo.br/pdf/ean/v15n2/ v15n2a04.pdf. [Acesso em 4 jan 2015].

40. Lazzeri G et al. Relationship between 8/9-yr-old school children BMI, parents' BMI and educational level: a cross sectional survey. Nutr J. 2011;19(10):76-82. Disponível em: . [Acesso em 15 set 2015].

41. Pergher RN et al. Is a diagnosis of metabolic syndrome applicable to children? O diagnóstico de síndrome metabólica é aplicável às crianças? J Pediatr Rio J. 2010; 86(2):1018. Disponível em: http://www.scielo.br/pdf/jped/v86n2/ v86n2a04.pdf. [Acesso em 3 jan 2014].

42. Leal VS et al. Excesso de peso em crianças e adolescentes no Estado de Pernambuco, Brasil: prevalência e determinantes. Cad Saúde Pública. 2012;28(6): 1175-82. Disponível em: http://www.scielo.br/scielo.php?script=sci_arttext\&pid=S0102-311X2012000600016\&lang=pt. [Acesso em 15 set 2015].

43. Momoi $\mathrm{C}$ et al. Saúde da criança: fatores de risco aplicados em programas de atençáo básica à saúde. Acta Paul Enferm. 2012;25(2):231-47. Disponível em: http://www.scielo.br/ pdf/ape/v25n2/a12v25n2.pdf. [Acesso em 4 jan 2014].

44. Sanches MS, Andrade RM. Percepciones y prácticas de madres de pre-escolares brasileños acerca de la alimentación de los hijos. Enferm. glob. 2011;10(21). Disponível em: http://scielo.isciii.es/scielo.php?script=sci_arttex$\mathrm{t} \& \mathrm{pid}=S 1695-61412011000100015$ \&lang=pt. [Acesso em 15 set 2015].

45. Marion Hare et al. Methods and baseline characteristics of a randomized trial treating early childhood obesity: The Positive Lifestyles for Active Youngsters (Team PLAY) trial. Contemp Clin Trials. 2012;33(3):534-49. Disponível em: http://www.ncbi.nlm.nih.gov/pmc/articles/PMC3336873/ pdf/nihms360469.pdf . [Acesso em 15 set 2015].

46. Naidu BM et al. Overweight among primary school-age children in Malaysia. Asia Pac J Clin Nutr. 2013;22(3):40815. Disponível em: http://www.nejm.org/doi/pdf/10.1056/ NEJMp1310577. [Acesso em 4 jan 2014]. 